\title{
Non-contact Characterization of Flexible Hybrid Electronics by Synchronized Thermography
}

\author{
Kari Remes \\ Optoelectronics and Measurement \\ Techniques Research Unit \\ University of Oulu \\ Oulu, Finland \\ kari.remes@oulu.fi
}

\author{
Tapio Fabritius \\ Optoelectronics and Measurement \\ Techniques Research Unit \\ University of Oulu \\ Oulu, Finland \\ tapio.fabritius@oulu.fi
}

\begin{abstract}
Eddy current heating with synchronized thermography (ST) is utilized for the contactless characterization of flexible hybrid electronics. A proposed approach is used for analyzing the uniformity of large area electronics being the basis for the quality assurance of hybrid electronics manufacturing. Flexible polymer substrate with printed conductors, bonded conventionally manufactured light-emitting diode (LED) chips and current regulators were used as test samples. Obtained results show that ST with eddy current heating is an effective and roll-to-roll compatible measurement tool for in-situ quality monitoring of hybrid electronics manufacturing.
\end{abstract}

Keywords - infrared imaging, uniformity, eddy-current, optical measurement techniques, printed electronics, quality control

\section{INTRODUCTION}

Recent progress in electronics materials and fabrication technologies have enabled the implementation of systems and technology platforms which haven't been available before. Novel features (flexibility, stretchability, transparency, and lightness) are providing plenty of attractive possibilities in various fields of applications [1] such as internet of things (IoT), wearables, autonomous vehicles, robotics and healthcare. Terms flexible electronics, printable electronics, organic and large area electronics etc., are used to distinguish this technology from conventional silicon-based electronics. In this context, the implementation of flexible or even conformable lighting and signage elements which can be embedded as a solid part of the structural elements have drawn great interest especially in automotive and architecture industry since it provides more freedom for the designers. The technological maturity level of flexible electronics is however limited and thus conventionally fabricated light emitting diode (LED) chips, bonded on printed flexible substrates, are mainly used in this type of products currently [2]. In this paper, the combination of flexible electronics with conventional electronics is called flexible hybrid electronics (FHE).

Once new materials and processes are utilized, manufacturing process upscaling with a high yield becomes an issue and leads to ambitious challenge for characterization and quality assurance technologies [3] [4]. The level of characterization as a function of the number of known parameters can be used as a measure for evaluating the maturity level of technology (see Fig. 1.). If the number of unknown parameters is large like in the case of FHE, there are exploitation bottlenecks which can be solved by intensive characterization. Due to the nature of FHE concept (large area, unusual material combinations, continuous roll-to-roll (R2R) manufacturing, etc.), the existing measurement techniques are not capable of providing all the necessary data for upscaling and optimization of the manufacturing processes of FHE. That is why novel in-situ quality monitoring tools, preferably capable for real-time monitoring, are needed axiomatically. It is worth to mention

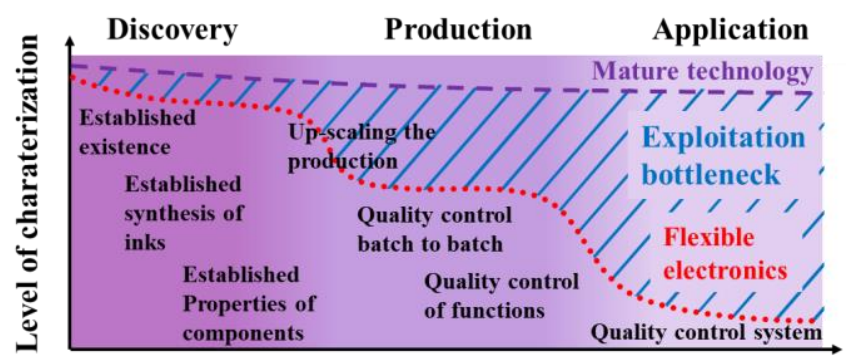

Number of known parameters

Fig. 1. Level of characterization as a function of known parameters. If the level of characterization is low, the exploitation of technology becomes challenging. This exploitation bottleneck can be solved by measurement technology. 
that due to the variation of maturity level of different process phases in FHE manufacturing chain, the need for different types of characterization is varied. Many aspects of the conventionally manufactured LED chips for example are well known, having thus significantly less need for characterization. However, the combination of LED chips on different materials is less known and thus the importance of material related aspects cannot be completely ignored in any phases of manufacturing.

The FHE technology is already emerging to the market but the existing quality assurance systems are not sophisticated. For example, the electrical performance testing and quality monitoring is mostly done for the end products only, being problematic for the optimization of the production yield. The great FHE specific challenges of quality assurance are that FHE products are typically large and that they contain structures which have at least one physical dimension in nanoscale. The high measurement coverage of large area samples with nanometer resolution is a difficult problem to be solved. The large number of different characterization technologies, based on various measurement principles, have been utilized in flexible electronics and FHE related research and product development [5]. To avoid an unnecessary extension of this paper, only a brief description of electronics property characterization is provided, since the proposed concept is primarily dedicated for that purpose. It is worth to notice that when the electrical properties such as conductivity of thin film structures are measured, the conductivity is not only depending on the material itself, but also on the physical size and morphology of the conductor. For example, the conductivity of a printed silver conductor is much less than that of a bulk silver due to the residuals and imperfect sintering of silver particles [6] [7].

Most common techniques dedicated to the electrical property testing of FHE are based on conductivity measurements with galvanic contact [8] [9]. The disadvantage of this type of methods is that they are time consuming if high lateral accuracy is needed. In addition, they require physical contact with the sample which might cause some defects and contaminations to electronics. If that approach is used in high volume in-situ measurements in FHE manufacturing, the measurements must be performed with 'stop and go' principle, which means that the production or testing line has to be stopped while the measurements are performed. Another well-known method is so called eddy current sensing (EC) [10] which consists of two key elements: an electronic oscillator and a coil (or a pair of coils). The oscillator induces an alternating current to the coil which generates a primary magnetic field. Once the conductive sample is interacting with the primary magnetic field, an electrical current is generated in the sample (so called eddy current) generating a secondary magnetic field which can be detected by the coil. The received signal is influenced by the properties of the sample and thus the electrical conductivity of the thin films can be evaluated. In case of thin metal deposition (including printing), grain sizes, contamination and defects can affect the overall conductivity of the layer, and that is why the conductivity is assumed as constant generating uncertainty to the measured absolute value. It is also so good to keep in mind that the lateral resolution of the eddy current approach is depending on the size of the used coils being typically in the range of some centimeters. However, the method is rather robust and insensitive to ambient conditions making it an interesting approach for FHE product in-situ quality monitoring. In addition, the Kelvin probe method, which is based on a vibrating capacitive device, has been used for contactless non-destructive work function monitoring of thin film solar cells [11].

Optics-based methods have shown to enable the non-destructive in-situ quality monitoring and the combination of infrared (IR) imaging with electrical heating is demonstrated to be an effective method for thin film large area electronics uniformity characterization. One of these methods is so called lock-in thermography where infrared (IR) imaging is synchronized with modulated electrical heating to analyze the temperature variation of the samples [12]. However, this technique is unnecessarily complex making it impractical to be utilized in real in-situ production environments. A simplified version of lock-in thermography is synchronized thermography (ST), which is also an IR imaging-based characterization method [13]. In that case, the sample is heated with direct current (DC) power and synchronized with IR imaging to produce thermograms, which are influenced by the non-uniformities of conductive thin films. Non-uniformities on electrical conductivity will influence on the 
current density thus modulating the temperature profile. The main advantage of this approach is that required data processing is very simple for identifying the defect locations and categories. Previous papers have shown that ST is a versatile technique to characterize the uniformity of thin films structures, providing quantitative information about the conductivity in R2R environment [14] - [18]. However, those measurements are based on electrical heating with galvanic contact to the sample being thus problematic. One important technology to be mentioned in this context is so called eddy current thermography which is a nondestructive testing technique combining eddy current heating $(\mathrm{ECH})$ and thermography to perform the structural characterization of electrically conductive materials such as metals [19] [20]. Induced eddy current is used to heat the tested object and the changes of induced eddy currents flows, indicating structural non-uniformities, are analyzed by a thermal camera. Recently, similar type of approach has been utilized also for electrical property uniformity characterization [21].

In our previously published paper, we used ST technology to characterize FHE samples where the powering wires were screen printed on a polymer substrate and conventionally fabricated regulators and LED chips were bonded on it [22]. In this extended version of the paper, similar FHE samples are characterized by ST but the measurements are done in a contactless manner. The capability of this approach to in-situ the R2R characterization of FHE samples is evaluated for the first time.

The structure of this paper is as follows: Section II describes the materials and methods, Section III presents the measurement results, Section IV discusses the benefits and issues of the technique, and finally Section V concludes the paper.

\section{MATERIALS AND METHODS}

\section{A. Flexible Hybrid Electronics test sample}

Two versions of FHE samples were measured as test samples (see Fig. 2.). The first sample is a kind of intermediate product, which has only $\mathrm{R} 2 \mathrm{R}$ printed silver conductors (Asahi 411AW) and dielectrics (Electrodag 452SS and Electrodag PF455B) without any surface mounted components. Polyethylene terephthalate (PET) with $125 \mu \mathrm{m}$ thickness was used as a substrate (Melinex ST506). This sample was used to characterize printed electronic structures without the influence of surface mounted components. Another sample type has LED chips (Luxeon

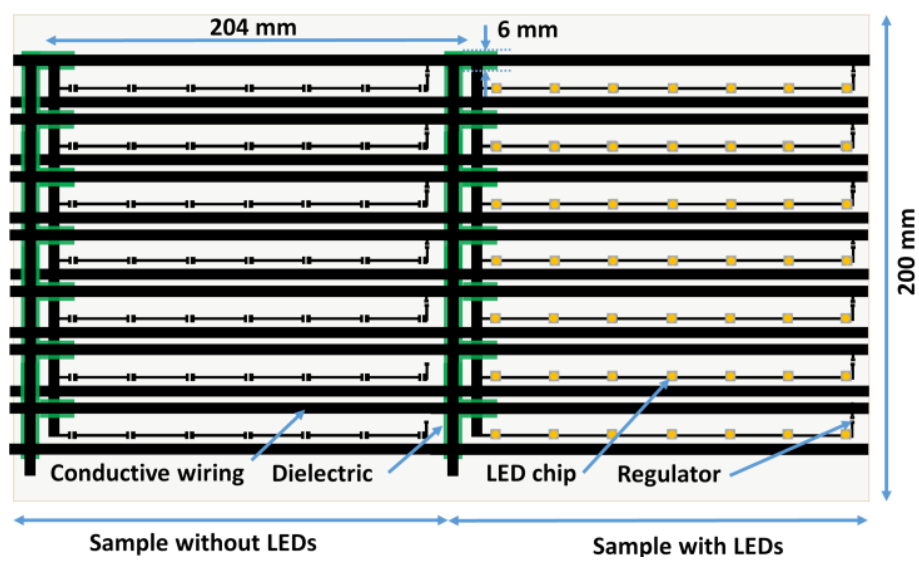

Fig. 2. Schematic layout of the measured flexible electronics sample. Sample without LEDs and sample with LEDs. 3535L) and regulators (NSI45030A, ON Semiconductor), which are bonded on the printed foil with adhesive glues (Epotek H20E and Loctite AA 3525). The layout of the electronics was designed to be compatible with R2R screen printing and mass customization. More detailed description of the sample design and manufacturing process is available in a previously published paper [2].

\section{B. Synchronized Thermograhy and Eddy Current Heating Based Measurement System}

A PI 640 IR camera (Optris GmbH) was used to capture IR images. The camera is based on a two-dimensional non-cooled focal plane array (UFPA) detector capable of measuring the IR emission of the heated object. The temperature measurement range covers $-20{ }^{\circ} \mathrm{C}$ to $900{ }^{\circ} \mathrm{C}$ in spectral range of $7.5-13 \mu \mathrm{m}$. The camera resolution is $640 \mathrm{x} 480$ pixels with $32 \mathrm{~Hz}$ frame rate. The lens of the camera has $33^{\circ} \times 25^{\circ}$ field of view (FOV) and the thermal sensitivity of the camera is $0.075 \mathrm{~K}$. The material and 
measurement conditions specific characteristic emissivity is adjustable between 0.100 and 1.000 . The IR camera has manual focus.

A microprocessor-controlled inductive heater (eddy current heating) CEIA PowerCube 32/900 (available frequency range 750 $\mathrm{kHz}-1150 \mathrm{kHz}$, maximum absorbed power $2.8 \mathrm{~kW}$ ) and a heating head HH10 equipped with an inductor coil (a simple one loop coil) was used to induce current to the printed conductors. More detailed theoretical explanation of heat induction in metallic structures is described by Tian et. al. [20]. The frequency is automatically chosen by the heat generator itself within the available range. The test samples were mounted on a PET carrier film which was enabling the measurement in an in-house made R2R testing device. The schematic of the used measurement setup is illustrated in Fig. 3. The power of the ECH heating unit was adjusted with the speed of the web in the used R2R device to heat up the conductive structures reasonably so that most of the conductive features of the sample become visible. Basically, the exposure time of sample to the inductive heating, is directly proportional to the speed of web. In this experiment, the speed of the web was $2 \mathrm{~m} / \mathrm{min}$ and the ECH power was $20 \%$ of the maximum power of the heater unit. Due to the limited size of the used inductive heating unit probe (limited by the capacitance of unit probe), each LED with conductive wiring in proximity were measured separately. Each measurement was performed 5 times and the location of the heating head was moved to the next position in the pattern. That was done because the length of the heating coil $(43 \mathrm{~mm})$ was much smaller than the measured web width $(200 \mathrm{~mm})$. It is worth to mention that this is not actually the limitation of the proposed approach and is simply caused because of the non-optimization of the inductive heating coil for this type of samples. The obtained raw IR image data were exported from the PI Connect software to Matlab for post-processing and analysis.

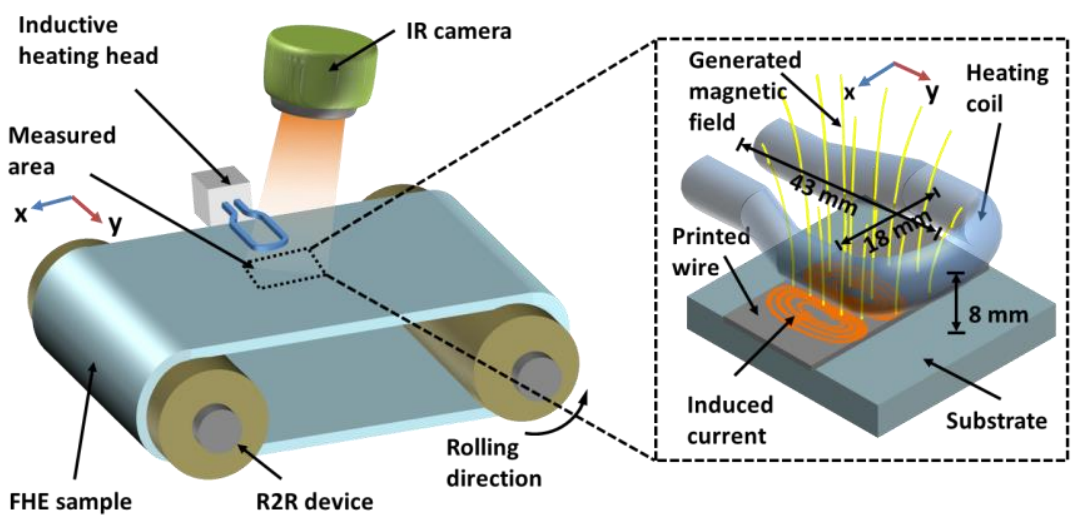

Fig. 3. The schematics of the used contactless inductive heating based R2R characterization setup. The power of induced current and local distribution of it is defined by the shape of the probe, and its distance to the sample.

Since this paper is a technical extension of our previously published paper [22], some of the earlier published results are shown also in this paper as a reference. However, the previously used measurement parameters and used measurement setup are not described here but the details can be found in the reference [22].

\section{Data processing}

To perform the comparative analysis of FHE samples during the inductive heating, a simple image processing algorithm was implemented in Matlab. Captured IR images in 8-bit grayscale format were processed similarly as in Ref. [21] to make temperature profile images suitable for the human eye to analyze. The data processing procedure was performed in the following way: 1) the captured IR image sequence (a video) of a full-length sample was stored to a temporary 3D image matrix; 2) a region of interest (ROI) of 1 x 330 pixels was selected at the same location (right after the highest temperature heating pattern as shown in Fig. 4) on each frame of the temporary matrix; 3) the ROI was handed over to another matrix for compiling of a temperature 
map of a sample (ROI by ROI); 4) a full-length IR image of the sample is formed after all line-shaped ROIs are stitched together; 5) the IR image is pseudo-colored to make temperature variation more visible. A line-shaped ROI was chosen to avoid issues with possible emissivity changes caused by the variation of the angle of view.

In this study, the required data analysis was done afterward and hence the term in-situ characterization means the capability to acquire all the necessary information from the manufacturing line in realistic R2R manufacturing conditions without data processing in this context. However, due to the simplicity of the used algorithm, the implementation of a real-time quality control system is most likely possible but not in the scope of this paper.

\section{MEASUREMENT RESULTS}

\section{A. Inductive heating profile analysis}

In the case of eddy current heating, the properties of the heating coil are known to have direct influence on the inducted current distribution on the sample. To investigate the heating profile of the used configuration, a uniform indium tin oxide (ITO) thin film on PET (Solutia - CPFilms Inc) was used as a test sample. The measurement conditions were adjusted to be comparable with the other measurements reported on this paper (See Fig 3.). The Obtained thermography image is shown in Fig. 4. The shape of the pattern correlates well with the shape of the heating coil. It is not uniform over the ROI used in the uniformity analysis of FHE (See the position of ROI in the Fig. 4.). Once the FHE samples are

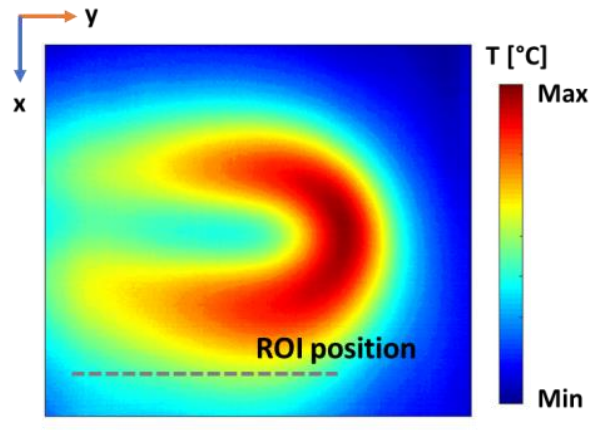

Fig. 4. The heating pattern of the induction coil and the position of the line-shaped ROI (grey dashed line) beside it. measured in-situ, the printed conductor has gone through the whole heating pattern before the ROI. In the R2R manufacturing, the same pattern (defined by the diameter of printing cylinder) is repeated (See the pattern in Fig. 2.) which means that all the patterns are exposed identically enabling the electrical uniformity analysis of FHE samples. However, the uniformity analysis within the same pattern is much more complicated.

\section{B. Measurement repeatibility analysis}

Once the electrical heating is done by contactless manner, the repeatability of measurement was evaluated. Each row of the matrix with LEDs was measured 5 times. The qualitative summary of representative row ( $4^{\text {th }}$ row) is depicted in Fig. 5.

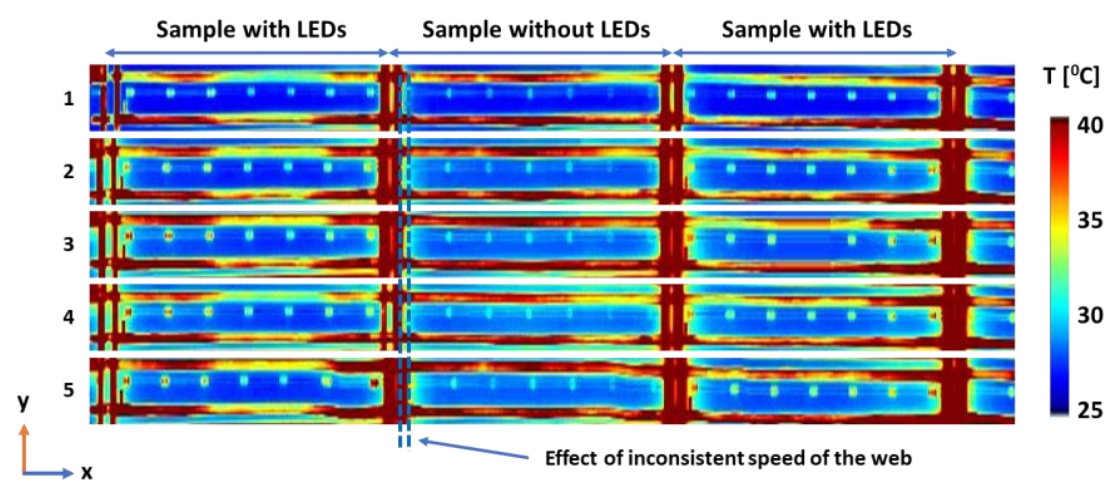

Fig. 5. Repeatability measurement of EC-heated structure with and without LEDs. The IR images of five subsequent measurements of the same structure are shown. Temperatures of these measurements from the same location are correlating well. However, due to the instability of sample movement during the in-situ measurement and possible fluctuation of the used magnetic field, some variation can be seen.

As seen in Fig. 5, the temperature profiles of all measurements are correlating well, indicating the good repeatability of measurement. However, some distortions can be observed. For example, the length of each section of the pattern is not equal 
(the lowest profile seems to have a longer section in the beginning) even though the actual physical length is not changed during the measurement. This distortion is explained by the inconsistent speed of the web during the measurement. From the uniformity characterization point of view, this might not be an issue, but has to be taken into account for further development of data processing. This measurement reveals another feature of ECH based measurement, which is not seen in our previous measurements [22]. The conductive lines perpendicular to the web's moving direction are having higher temperatures than the parallel ones. Some parts of the temperature profiles of the conductors are saturated on these regions. This can be explained by the stronger electromagnetic interaction of the conductor instead of significant difference in electromagnetic or thermal properties of the printed structures. As explained before, this was not a problem in pattern-to-pattern uniformity analysis, due to the nature of roll-to-roll manufacturing. Printing machine has a certain repetition length defined by the diameter of a printing cylinder. This means that the same pattern is repeated during the manufacturing process. From uniformity point of view, the manufacturing line operator is interested in the similarity of repeated patterns. Even though our inductive heating pattern is not optimal due to the shape of existing heating coil, our experimental results show good repeatability (Fig. 5). This means that if the printing quality has changed for some reason, the obtained thermography images of adjacent printing patterns are not identical and can be thus used as a measure for the uniformity of manufactured electronics.

\section{Uniformity analysis of conductors and dielectrics}

Our previous ST measurement showed that the majority of the printed conductive structures have significant non-uniformities in their electrical conductivity [22]. All the seven rows of the sample with the surface mounted LEDs and regulators were measured by ST with ECH to investigate the possible differences in contrast to the galvanic contact based electric heating. Comparison of the temperature profiles obtained by different heating methods is depicted in Fig. 6.

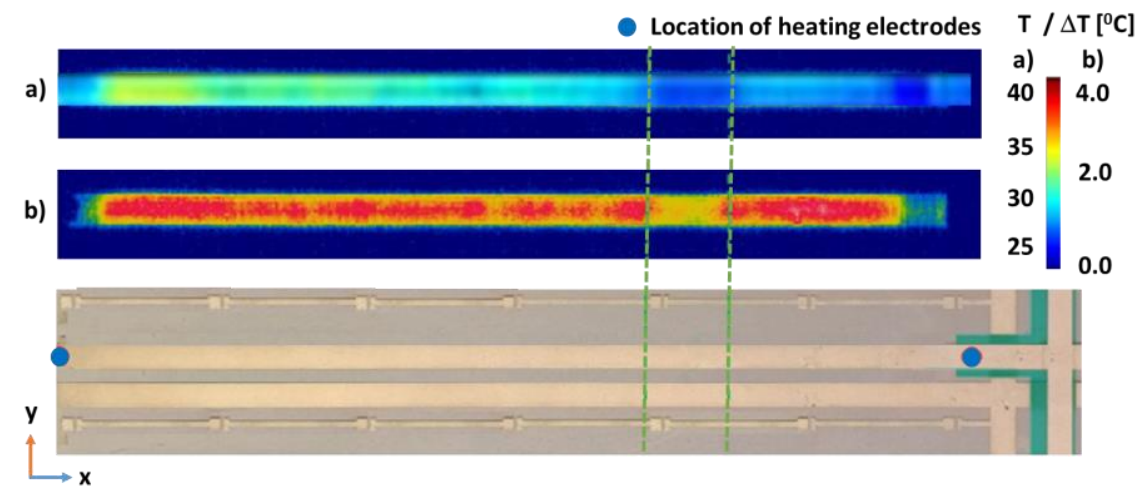

Fig. 6. Uniformity analysis of a printed conductive line based on a) ECH heating and b) galvanic contact based heating [20]. Good correlation of the temperature profiles obtained with different methods is observed. Due to the different heating conditions, the scale of the pseudo-colored temperatures profiles in a) and b) are different. Profile a) has much higher absolute temperature elevations.

In contrast to electrical heating with galvanic contact, the quantitative analysis of conductivity non-uniformities becomes more complicated when ECH is used since the electromagnetic wave interaction with the printed structures is influenced by additional factors such as the distance of the printed line from the heating coil and possible non-uniformities of the magnetic flux. In case 
of eddy current heating, also the skin-effect may influence on current generation, having impact on temperature profiles obtained by ST.

The uniformity and structural integrity of dielectric layers in printed LED foils are important for the device functionality point of view because the unevenness or defects in dielectric layers could cause breakdowns when powered electrically. The dielectric layer is partly sandwiched between the conductive lines thus limiting the area which can be characterized by the ST methods. Conductive lines beneath and above of the dielectric were heated by eddy current and temperature profiles were captured by ST. Obtained results were compared with the corresponding profile of the electrically heated conductor with$$
\text { colvanic contact [22]. The obtained results are shown in Fig. 7. Since the }
$$
printed conductors that area has smaller resistivity. In the Fig. 7 a), the effect of double thickness is clearly seen in the ST image captured during the galvanic contact heating however, whereas in case of ECH this effect is difficult to distinguish. In the case of $\mathrm{ECH}$, the highest temperature is observed in the single print area (between the brown dashed lines) being mostly influenced by the fact that conductive line is parallel to the heating coil. The heat generation in the other parallel line (between dashed brown and blue lines) is much less. This is an indication that the dielectric printing modifies the conductivity of printed silver beneath (and in proximity). This observation requires further investigation to be confirmed but is most probably related to the interaction of printed silver and the solvent of dielectric ink.

\section{Conductive wiring structural analysis}

The electrical conductivity of printed conductors is known to depend on the various parameter including the physical dimensions. Our previous measurement proved that ST can distinguish the thickness variation when the galvanic contact based heating is used [22]. However, in case of ECH, this type of analysis becomes more challenging since the heat induction is more sensitive to the heating conditions. Without having prior knowledge of the sample structure, ECH-based ST measurement seems not to be very reliable for the structural analysis within the same pattern of the printed conductors even though similar temperature variations can be observed.

\section{E. Effect of heating arrangement}

As seen in Fig. 5, the use of a non-optimized heating coil causes uneven heating of the conductive structures. To demonstrate the effect of a heating head arrangement on the IR imaging, the heating coil was set in three alternative positions as shown in Fig. 8. The power of ECH unit has to be raised to $60 \%$ of the maximum power to achieve a reasonable heating of the sample structures when the coil is in the tilted position. The tilted coil arrangement (IR image shown in Fig. 8. a) seems to emphasize mainly traverse (y-direction) structures whereas the rotated coil arrangement presented in Fig. 8. c) heats some lengthwise (x-direction) features more efficiently. As stated earlier, it is always vital to have in-depth knowledge of the sample

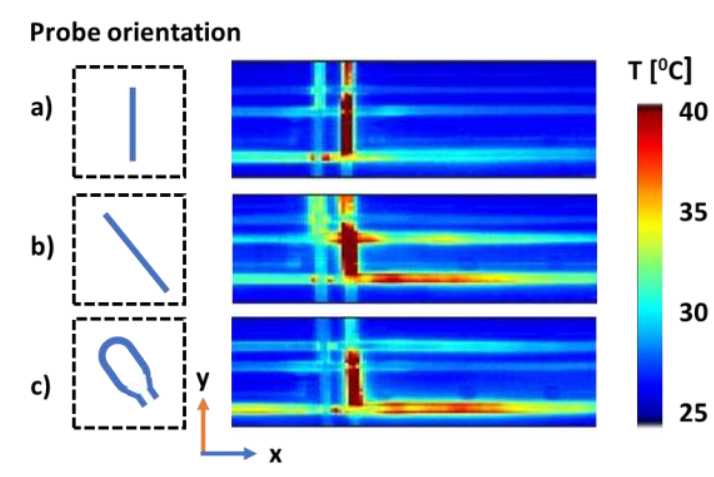

Fig 8. Effect of the heating coil arrangement on IR imaging. The same detail of the sample with three different heated arrangements are shown. a) Coil tilted $90^{\circ}$, b) Coil rotated $40^{\circ}$ and tilted $90^{\circ}, \mathrm{c}$ ) Coil rotated $40^{\circ}$ counter-clockwise, 
structures to do useful analysis of the results and to optimize the heating arrangement for the structure in question.

\section{F. Defect detection}

The ST method is not selective for different types of unevenness.

Similar temperature elevations can be observed even though the origin of it is different. Basically, this means that the local temperature gradient is a clear indication of the unevenness, sometimes even a defect. To demonstrate the influence of defect, some part of a conductor was removed by a knife thus reducing the cross-sectional area. A clearly visible hot-spot with significant temperature elevation can be seen above the crack (see Fig. 9) regardless the heating method. The result here discloses that any significant localized temperature increase

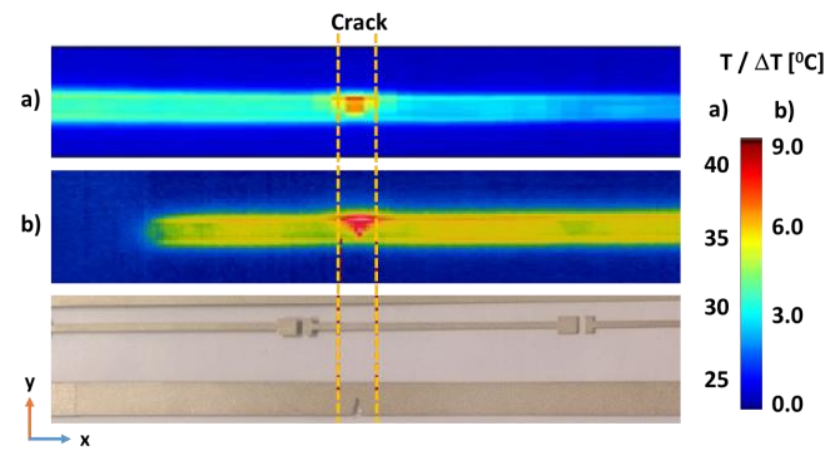

Fig. 9. Visually observable crack in a conductive line and its characterization by ST heated by a) ECH and b) galvanic contact [22]. should be considered as a major defect while the absolute temperature rise limit is depending on the heating conditions.

\section{G. LED chip characterization}

From the LED matrix manufacturing yield point of view, the in-situ characterization of the surface mounted LED chips themselves is important. Once the galvanic contact based electrical heating is used, the LEDs can be characterized with some extent by ST [22]. Once the ECH is used, the heating of LED chips can be also observed as shown in Fig. 10. However, the temperature profiles of LEDs vary significantly and more detailed quantitative analysis were not done. Reliable LED chip functionality characterization requires further work in terms of optimization of ECH conditions.

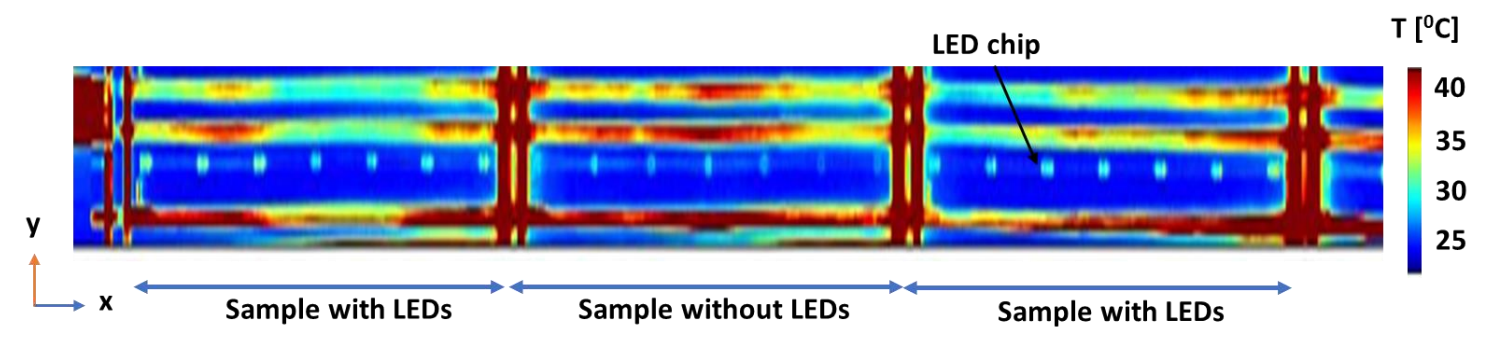

Fig 10. LED Characterization by ECH heating (the measurement data corresponds to the data set 1 in the Fig. 5). Once the distance and the location of the heating coil is correct, clear heat generation in LED chips can be observed. However, large variation of temperature elevation is obvious. Without heating conditions optimization, LED performance or functionality characterization by ECH-based ST is difficult.

\section{DISCUSSIONS}

The obtained results of the ST measurements based on ECH are encouraging and mostly consistent with expectations. Performed measurement results show that larger sample areas of thin film electronics with bonded components can be characterized by contactless and R2R compatible way, nicely complementing the existing characterization techniques dedicated to this type of measurements. On the other hand, observed results raise several questions which need further investigation.

ECH-based ST is sensitive to identifying the surface discontinuities in printed silver conductive lines and the measurements provided spatial information on the current density distribution in them. In contrast to galvanic contact based heating, the quantitative analysis is more demanding because the local heat generation depends on the physical structure of the printed pattern and heating head arrangement. For example, the direction of the conductor had strong influence on the heating even though the absolute electrical conductivities of those lines were not varying significantly. At the same time, parameters such as the shape of the induction coil and the distance between the coil and the sample had high impact on heat generation. These shortcomings can 
be most probably solved by optimizing the heating conditions and performing simultaneous measurements in different directions at the same time.

Possibilities for the heating coil design optimization were limited due to the electronics of the inductive heating unit available. The inductance of the heating coil has to be matched with the capacitance of the heating unit to achieve a proper magnetic field for the eddy current heating. Thus, the length of the heating coil was restricted (maximum length used in the experiments), making impossible to investigate some interesting coil designs (long enough coil to cover the whole width of the sample) in this setup. However, this is not the limitation of ECH concept, but the restriction of a specific heating unit used in this study.

All the experiments were done so that heating coil and IR camera were on the same side of the sample. Alternative setup could be based on the arrangement where heating head is beneath the investigated sample and IR camera is on the other side. That would enable more freedom to implement measurement a system which can be installed in R2R manufacturing lines without the extensive modifications of the line itself. It is worth to mention that in printed electronics R2R manufacturing lines, the free space available for additional measurement systems can be very limited.

One aim of this study was to analyze the functionality of conventional electronics based surface bonded components on flexible substrate. Once the LEDs can be distinguished (See Fig. 10.), most probably some aspects of their functionality can be analyzed by ECH based ST. That information is valuable when the quality assurance of LED foil R2R manufacturing is developed. One of those parameters are the quality of bonding or misbehavior of a LED which should cause temperature elevation in the LED or its proximity. Due to the large variation on heating, any confirmed conclusions about their functionality were hard to make. All the aspects on influencing that phenomena are not clearly understood and require further investigation.

ECH increases the complexity of data analysis but it still has major advantage over the galvanic contact based heating because it does not require physical contact with the sample. This is extremely important especially in case of thin film electronics where many of the materials are easily damaged by any physical contact with them. Another interesting aspect of the ECH based method is that the eddy current induction in the material can be controlled by the frequency of heating coil modulation. Once the frequency of the heating magnetic field is changed, the eddy current characteristics in the medium are different. Varying the frequency of the eddy current heating would enable a spectroscopic approach to electrical property characterization. In contrast to the conventional eddy current electronics characterization [23], the lateral resolution of the ECH-based ST doesn't depend on the size of the heating probe making it superior to a conventional eddy current method.

Once the data analysis is more demanding in the case of ECH heating, an interesting approach to analyze ST data in more details could be the combination of ST measurements and the simulation of electrical and thermo-mechanical characteristics of FHE products such as the LED foil described in this study. That approach would increase the confidence of the data analytics obtained by ECH-based ST. Preliminary work related to FHE simulations has already started [24] and it is expected also to deepen the understanding about the reliability of flexible, large area electronics.

\section{CONCLUSION}

Demonstrated R2R compatible contactless electrical characterization system is simple and fast in comparison to other known methods used in printed electronics and FHE in-situ characterization making proposed approach superior for quality monitoring of FHE production in R2R. The repeatability of the measurement was good making the in-situ electrical uniformity analysis of adjacent patterns possible. However, the uniformity analysis within the same pattern becomes more complex and requires further development. It is also worth mentioning that the proposed concept enables easy adjustment of the measuring area, making the method promising especially for large area electronics applications. Proposed concept was tested for samples with 
printed conductive lines and surface mounted LED components, but it is expected to work also for other type of electrically functional structures and components such as antennas, sensors, transistors, solar cells etc. Described setup is not optimized for FHE and thus further analysis and system and data processing algorithm development are needed to exploit the potential of this technology not only for uniformity analysis but also for defect localization and classification. In addition, a possibility to modulate the frequency of inductive heating enables a sort of spectroscopic analysis of electronic materials.

\section{ACKNOWLEDGMENT}

We thank Technical Research Center of Finland Ltd (VTT) for providing the LED foil samples and technical advice for this research. Authors would also like to thank the financial support from the Academy of Finland's FIRI funding (grant no. 320017) and EU Regional Development Fund (A74080).

\section{REFERENCES}

[1] W. Wu, "Inorganic nanomaterials for printed electronics: a review," Nanoscale, vol. 9, no. 22, pp. $7432-7372,2017$.

[2] K. Keränen, P. Korhonen, J. Rekilä, O. Tapaninen, T. Happonen, P. Makkonen and K. Rönkä, "Roll-to-roll printed and assembled large area LED lighting element," International Journal of Advanced Manufacturing Technology, vol. 81, pp. 529-536, 2015.

[3] E.K.Richman and J.E.Hutchison, "The nanomaterial characterization bottleneck," ACS Nano, vol. 3, no. 9, pp. 24412446, 2009.

[4] E. Masnke, G. Jäger, T. Hausotte and R. Füß1, "Recent developments and challenges of nanopositioning and nanomeasuring technology," Measurement Science and Technology, vol. 23, 2012.

[5] R. Tone and S. Ganz, "Characterization techniques for printed electronics," in Organic and Printed Electronics, Singapore, Pan Stanford Publishing Pte. Ltd., 2016, pp. 118-146.

[6] J. Perelaer, R. Jani, M. Grouchko, A. Kamyshny, S. Magdassi and U. S. Schubert, "Plasma and Microwave Flash Sintering of a Tailored Silver Nanoparticle Ink, Yielding 60\% Bulk Conductivity on Cost - Effective Polymer Foils," Advanced Materials, vol. 24, pp. 3993-3998, 2012.

[7] E. Hannila, B. Augustine, T. Kurkela, J. Lauri and T. Fabritius, "Yield and Electrical Functionality of the Glass Laminated Conductive Wires and Connectors," IEEE Transactions on Components, Packaging and Manufacturing Technology, 2019.

[8] K.Shimanovich, Y. Bouhadana, D. A. Keller, S. Rühle, A. Y. Anderson and A. Zaban, "Four-point probe electrical resistivity scanning system for large area conductivity and activation energy mapping," Review of Scientific Instruments, vol. 85, p. 055103, 2014.

[9] T.Happonen, T. Kokko, E. Juntunen and K. Rönkä, "Quality assurance for rotary screen printed wiring backplanes with automated roll-to-roll electrical test equipment," Flexible and Printed Electronics, vol. 4, no. $2,2019$.

[10] H. Heuer, S. Hillmann, M. Roellig, M. Schulze and K.-J. Wolter, "Thin Film Characterization using High Frequency Eddy Current Spectroscopy," in Electromagnetic Nondestructive Evaluation (XIII), 2010.

[11] B. Van Aken, K. Bakker, M. Heijna, D. Reid, I. Baikie and W. Soppe, "In situ measurements of surface (photo)voltage of roll-to-roll deposited thin film silicon solar cells," Physica Status Solidi, vol. 207, pp. 682-685, 2010.

[12] J. Rakotoniaina, O. Breitenstein and M. Langenkamp, "Localization of weak heat sources in electronic devices using highly sensitive lock-in thermography," Materials Science and Engineering B, Vols. 91-92, pp. 481-485, 2002.

[13] K. Leppänen, J. Saarela, R. Myllylä and T. Fabritius, "Electrical heating synchronized with IR imaging to determine thin film defects," Optics Express, vol. 21, pp. 32358-32370, 2013.

[14] K. Leppänen, J. Saarela and T. Fabritius, "IR-imaging based system for detecting the defects of conductive materials," in Proc. 2014 SPIE Optical Engineering + Applications, Vol. 9205, 92050M., San Diego, 2014.

[15] K. Leppänen, J. Saarela and a. T. Fabritius, "Synchronized thermography for multilayer thin film characterization," in Proc. SPIE Thin Films for Solar and Energy Technology VI, Vol. 9177, 917705, San Diego, 2014.

[16] C. Schuss, K. Leppänen, K. Remes, J. Saarela, T. Fabritius, B. Eichberger and T. Rahkonen, "Detecting Defects in Photovoltaic Cells and Panels and Evaluating the Impact on Output Performances," IEEE Trans. Instrum. Meas., vol. 65, no. 5, pp. 1108-1119, 2016.

[17] J.-V. Voutilainen, T. Happonen, J. Häkkinen and T. Fabritius, "All silk-screen printed polymer-based remotely readable temperature sensor," IEEE Sensors Journal, vol. 15, no. 2, pp. 723-733, 2015.

[18] K. Remes, K. Leppänen and T. Fabritius, "Thermography based online characterization of conductive thin films in large-scale electronics fabrication," Optics Express, vol. 26, no. 2, pp. 1219-1229, 2018.

[19] N. Tsopelas and N. Siakavellas, "Experimental evaluation of electromagnetic-thermal non-destructive inspection by eddy current thermography in square aluminum plates," NDT\&E International, vol. 44, pp. 609-620, 2011.

[20] G. Tian, Y. Gao, K. Li, Y. Wang, B. Gao and Y. He, "Eddy Current Pulsed Thermography with Different Excitation Configurations for Metallic Material and Defect Characterization," Sensors, vol. 16, no. 6, pp. 843-853, 2016. 
[21] K. Remes, A. Järvenpää and T. Fabritius, "Contactless online characterization of large-area conductive thin films by thermography and induction," Optics Letters, vol. 44, no. 10, pp. 2574-2577, 2019.

[22] K. Remes, A. Latomäki and T. Fabritius, "Flexible electronics non-destructive uniformity characterization by synchronized thermography," in 2019 IEEE International Instrumentation and Measurement Technology Conference (I2MTC), Auckland, 2019.

[23] H. Hauer, S. Hillmann, M. Roellig, M. H. Schulze and K.-J. Wolter, "Thin film characterization using high frequency eddy current spectroscopy," in 2009 9th IEEE Conference on Nanotechnology (IEEE-NANO), Genoa, 2009.

[24] J. Lauri, E. Hannila and T. Fabritius, "Non-destructive characterization of glass laminated electronics," in 2019 IEEE International Instrumentation and Measurement Technology Conference (I2MTC), Auckland, 2019. 